\title{
Determination of elliptic curves with everywhere good reduction over real quadratic fields $\mathbb{Q}(\sqrt{3 p})$
}

\author{
by \\ TAKAAKI KagaWA (Kusatsu)
}

1. Introduction. Let $k=\mathbb{Q}(\sqrt{m})$ be a real quadratic field, where $m$ is a squarefree integer greater than 1 . In our previous papers [4] and [5], we determined all elliptic curves with everywhere good reduction over $k$ when $m=37$ and 29, respectively. In the course of the determination, we constructed some unramified abelian extensions by applying Serre's results (the corollary to Proposition 11 and Proposition 12 in [14]) to the field of 3 -division points. Unfortunately, we cannot apply them to the case $m \equiv$ $0(\bmod 3)$ because of their assumption. However, without them, we can construct certain abelian extensions unramified outside 3 and the infinite primes. Thus assuming certain conditions on ray class numbers, we can deduce some criteria, and using them we can treat the case $m \equiv 0(\bmod 3)$.

If $1<m<100, m \equiv 0(\bmod 3)$, and the class number of $k$ is prime to 6 , then $m=3,6,21,33,57,69$ or 93. In [5], [7], [9], the proof is given for the nonexistence of elliptic curves with everywhere good reduction over $k$ when $m=3,21$ and all such curves are determined when $m=6$, while the cases $m=33,57,69$ and 93 are still open. In this paper, we determine all elliptic curves with everywhere good reduction over $\mathbb{Q}(\sqrt{33})$ and show the nonexistence of such curves over $\mathbb{Q}(\sqrt{57}), \mathbb{Q}(\sqrt{69})$ and $\mathbb{Q}(\sqrt{93})$.

We use the following notation throughout this paper. For an algebraic number field $k, \mathcal{O}_{k}, \mathcal{O}_{k}^{\times}$and $h_{k}$ denote the ring of integers, group of units and class number of $k$, respectively. If $\mathfrak{m}$ is a divisor of $k$ (that is, a formal product of a fractional ideal of $k$ and some infinite primes of $k), h_{k}(\mathfrak{m})$ denotes the ray class number modulo $\mathfrak{m}$. If $k$ is a real quadratic field, then $\varepsilon$ and ' denote the fundamental unit greater than 1 and the conjugation of $k$, respectively.

For an elliptic curve $E$, we denote by $j(E)$ and $\Delta(E)$ the $j$-invariant and the discriminant of $E$, respectively.

2000 Mathematics Subject Classification: Primary 11G05. 
2. Results. Let $k=\mathbb{Q}(\sqrt{33})$. The fundamental unit of $k$ is $\varepsilon=23+$ $4 \sqrt{33}$. In [9], the following elliptic curve with everywhere good reduction over $k$ is given:

$$
E_{1}: y^{2}+(5+\sqrt{33}) x y+\varepsilon y=x^{3}, \quad \Delta\left(E_{1}\right)=-\varepsilon^{3}, \quad j\left(E_{1}\right)=-32768 .
$$

This curve contains two $k$-rational subgroups $V_{1}, V_{2}$ of order 3 , namely

$$
V_{1}=E_{1}(k)_{\text {tors }}=\langle(0,0)\rangle, \quad V_{2}=\left\langle\left(-6-\sqrt{33}, y_{1}\right)\right\rangle,
$$

where $y_{1}=(40+7 \sqrt{33}+\sqrt{-\varepsilon}) / 2=(40+7 \sqrt{33}+2 \sqrt{-3}+\sqrt{-11}) / 2$. Let $E_{2}:=E_{1} / V_{1}, E_{3}:=E_{1} / V_{2}$. Using Vélu's formula [18], we obtain the following defining equations of $E_{2}$ and $E_{3}$ :

$$
\begin{gathered}
E_{2}: y^{2}+(5+\sqrt{33}) x y+\varepsilon y=x^{3}-(1235+215 \sqrt{33}) x-(35915+6252 \sqrt{33}), \\
\Delta\left(E_{2}\right)=-\varepsilon, \quad j\left(E_{2}\right)=-(5+\sqrt{33})^{3}(5588+972 \sqrt{33})^{3} \varepsilon^{-1} \\
E_{3}: y^{2}+(5+\sqrt{33}) x y+\varepsilon y=x^{3}+(85+15 \sqrt{33}) x+(730+127 \sqrt{33}) \\
\Delta\left(E_{3}\right)=-\varepsilon^{5}, \quad j\left(E_{3}\right)=-(5-\sqrt{33})^{3}(5588-972 \sqrt{33})^{3} \varepsilon
\end{gathered}
$$

Although $j\left(E_{1}\right)=j\left(E_{1}^{\prime}\right)$ (resp. $j\left(E_{2}\right)=j\left(E_{3}^{\prime}\right)$ ), $E_{1}$ and $E_{1}^{\prime}$ (resp. $E_{2}$ and $\left.E_{3}^{\prime}\right)$ are not isomorphic over $k$, since $\Delta\left(E_{1}\right) / \Delta\left(E_{1}^{\prime}\right)=\Delta\left(E_{2}\right) / \Delta\left(E_{3}^{\prime}\right)=\varepsilon^{6}$ is not a 12 th power. Hence there are at least six $k$-isomorphism classes of elliptic curves with everywhere good reduction over $k$.

By definition, $E_{2}$ and $E_{3}$ are 3-isogenous over $k$ to $E_{1}$. Further we see that $E_{1}$ and $E_{1}^{\prime}$ are 11-isogenous over $k$, since $E_{1}$ and $E_{1}^{\prime}$ are quadratic twists by $-\pi_{11} / 11$ and $\pi_{11}^{\prime} / 11^{2}$ of the curves $121 \mathrm{~B} 1$ and $121 \mathrm{~B} 2$ in Table 1 of [2], respectively, 121B1 and 121B2 are 11-isogenous over $\mathbb{Q}$, and $\left(-\pi_{11} / 11\right)\left(\pi_{11}^{\prime} / 11^{2}\right)=1 / 11^{2}$. Here $\pi_{11}=11+2 \sqrt{33}$ is a prime element of $k$ dividing 11 . Below is the isogeny graph of the related elliptic curves:

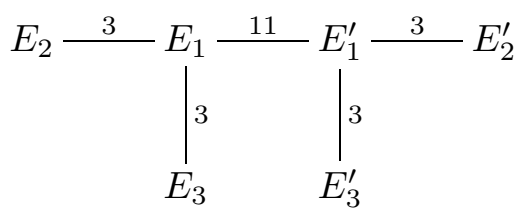

Here, for a prime $p$ and elliptic curves $E$ and $\bar{E}$ defined over $k$, the graph

$$
E \stackrel{p}{E}
$$

means that $E$ and $\bar{E}$ are $p$-isogenous over $k$. Hence there is at least one $k$-isogeny class of elliptic curves with everywhere good reduction over $k$.

In this paper we prove

TheOREM 1. Up to isomorphism over $k=\mathbb{Q}(\sqrt{33})$, the six curves listed above are all the elliptic curves with everywhere good reduction over $k$. In particular, there is exactly one $k$-isogeny class of such curves. 
We simultaneously prove the following theorem:

THEOREM 2. There are no elliptic curves with everywhere good reduction over $\mathbb{Q}(\sqrt{m})$ if $m=57,69$ or 93 .

Let $d$ be the discriminant of a real quadratic field and $\chi_{d}$ the Dirichlet character associated with $d$. Let $S_{d}=S_{2}\left(\Gamma_{0}(d), \chi_{d}\right)$ be the space of cuspforms of Neben-type of weight 2 and level $d$. It is conjectured (cf. [12]) that any elliptic curve having everywhere good reduction over the real quadratic field $\mathbb{Q}(\sqrt{d})$ and admitting an isogeny over $\mathbb{Q}(\sqrt{d})$ to its conjugate should be isogenous over $\mathbb{Q}(\sqrt{d})$ to so-called Shimura's elliptic curve which arises from a 2-dimensional $\mathbb{Q}$-simple factor of $S_{d}$. When $d=33,57,69,93$, it is known that $S_{d}$ is 2-dimensional and $\mathbb{Q}$-simple, 4-dimensional and $\mathbb{Q}$-simple, 6-dimensional and $\mathbb{Q}$-simple, 8-dimensional and $\mathbb{Q}$-simple, respectively. Thus Theorems 1 and 2 confirm the conjecture for these four values of $d$.

3. Preliminaries. Later we will give criteria for every elliptic curve with everywhere good reduction over a real quadratic field $k$ to admit a 3isogeny defined over $k$ (Propositions 11 and 12 below). Thus we first study elliptic curves with 3-isogeny and some diophantine equations arising from the investigation of such curves. Further, since a key tool to prove the criteria is the field $L=k(E[3])$ of 3 -division points and $\mathrm{Gal}(L / k)$ can be viewed as a subgroup of the general linear group $\mathrm{GL}_{2}\left(\mathbb{F}_{3}\right)$, we will also study subgroups of $\mathrm{GL}_{2}\left(\mathbb{F}_{3}\right)$.

3.1. Elliptic curves with 3-isogeny. Let $E$ and $\bar{E}$ be elliptic curves defined over a number field $k$ which are 3 -isogenous over $k$. We define a rational function $J(x)$ by

$$
J(x)=\frac{(x+27)(x+3)^{3}}{x} .
$$

Then, by Pinch [13], the $j$-invariants of $E$ and $\bar{E}$ can be written as

$$
j(E)=J(t), \quad j(\bar{E})=J(\bar{t}), \quad t, \bar{t} \in k, t \bar{t}=729=3^{6} .
$$

(This is nothing but a parametrization of the modular curve $Y_{0}(3)$.) Moreover, let $c_{4}(E)$ and $c_{6}(E)$ be the usual quantities associated with $E$. Then the following relations hold:

$$
\begin{gathered}
j(E)=\frac{c_{4}(E)^{3}}{\Delta(E)}=\frac{(t+27)(t+3)^{3}}{t}, \\
j(E)-1728=\frac{c_{6}(E)^{2}}{\Delta(E)}=\frac{\left(t^{2}+18 t-27\right)^{2}}{t} .
\end{gathered}
$$

Lemma 3. Let $k, E, \bar{E}, t$ and $\bar{t}$ be as above. Then

(1) If $j(E) \neq 1728$, then $t / \Delta(E)$ is a square in $k$. 
(2) If $E$ and $\bar{E}$ have everywhere good reduction over $k$ and $j(E), j(\bar{E}) \neq$ 0,1728 , then the principal ideals $(t)$ and $(\bar{t})$ are integral and sixth powers.

Proof. (1) follows immediately from (3.2).

(2) It suffices to prove the assertions only for $t$. Equation (3.1) and the assumption that $E$ has everywhere good reduction over $k$ imply that $t$ is an integer in $k$. By the same assumption, the principal ideal $(\Delta(E))$ is a 12 th power, say $(\Delta(E))=\mathfrak{a}^{12}$. Since $j(E) \neq 1728$, we see from (3.2) that $(t)=\left(\left(t^{2}+18 t-27\right) / c_{6}(E)\right)^{2} \mathfrak{a}^{12}$ is a square. To show that $(t)$ is a cube, it is enough to show that $\operatorname{ord}_{\mathfrak{p}}(t) \equiv \operatorname{ord}_{\mathfrak{p}}(27)(\bmod 3)$ for any prime ideal $\mathfrak{p}$ dividing 3 , where $\operatorname{ord}_{\mathfrak{p}}$ is the normalized valuation corresponding to $\mathfrak{p}$, since $t, \bar{t} \in \mathcal{O}_{k}$ and $t \bar{t}=3^{6}$. We may suppose that $\operatorname{ord}_{\mathfrak{p}}(t) \geq \operatorname{ord}_{\mathfrak{p}}(27)$. If $\operatorname{ord}_{\mathfrak{p}}(t)=\operatorname{ord}_{\mathfrak{p}}(27)$, then there is nothing to prove. If $\operatorname{ord}_{\mathfrak{p}}(t)>\operatorname{ord}_{\mathfrak{p}}(27)$, then $\operatorname{ord}_{\mathfrak{p}}((t+27) / t)=\operatorname{ord}_{\mathfrak{p}}(27)-\operatorname{ord}_{\mathfrak{p}}(t)$. On the other hand, since $j(E) \neq 0$, we see from $(3.1)$ that $((t+27) / t)=\left(c_{4}(E) /(t+3)\right)^{3} / \mathfrak{a}^{12}$ is a cube. Hence $\operatorname{ord}_{\mathfrak{p}}(t) \equiv \operatorname{ord}_{\mathfrak{p}}(27)(\bmod 3)$.

Let $k$ be a real quadratic field and let $E$ be an elliptic curve having everywhere good reduction over $k$ and admitting a 3-isogeny defined over $k$ with $j(E)=J(t)$. In this case, $j(E)$ is neither 0 nor 1728 (Theorem 2(a) in [16]). Thus it follows from Lemma 3(2) that
$(t)= \begin{cases}(1), & (729) \\ (1), & (27),(729) \\ (1), & \mathfrak{p}^{6}, \mathfrak{p}^{\prime 6},(729)\end{cases}$
if 3 is inert,
if 3 ramifies,
if $(3)=\mathfrak{p p}^{\prime}, \mathfrak{p}$ and $\mathfrak{p}^{\prime}$ are distinct prime ideals.

From (3.1), we have

$$
\left(\frac{c_{4}(E)}{t+3}\right)^{3}=\Delta(E)(1+27 u), \quad u=\frac{1}{t} \in \mathcal{O}_{k}^{\times}
$$

if $(t)=(1)$,

$$
\left(\frac{3 c_{4}(E)}{t+3}\right)^{3}=\Delta(E)(u+27), \quad u=\frac{729}{t} \in \mathcal{O}_{k}^{\times}
$$

if $(t)=729$, and

$$
\left(\frac{c_{4}(E)}{t+3}\right)^{3}=\Delta(E)(1+u), \quad u=\frac{27}{t} \in \mathcal{O}_{k}^{\times}
$$

if 3 is ramified and $(t)=(27)$. Suppose that 3 decomposes in $k$ as $\mathfrak{p p}^{\prime}$, that $(t)=\mathfrak{p}^{6}$, and that $\left(h_{k}, 6\right)=1$. Then $\mathfrak{p}$ is principal, say $\mathfrak{p}=(\pi), \pi \in \mathcal{O}_{k}$. From (3.1) we have

$$
\left(\frac{\pi c_{4}(E)}{t+3}\right)^{3}=\Delta(E)\left(\pi^{3} \pm \pi^{\prime 3} u\right), \quad u=\frac{\pi^{6}}{t} \in \mathcal{O}_{k}^{\times} .
$$

Similarly, if $(3)=\mathfrak{p p}^{\prime},(t)=\mathfrak{p}^{\prime 6}=\left(\pi^{\prime 6}\right)$, then 


$$
\left(\frac{\pi^{\prime} c_{4}(E)}{t+3}\right)^{\prime 3}=\Delta(E)^{\prime}\left(\pi^{3} \pm \pi^{\prime 3} u\right), \quad u=\frac{\pi^{6}}{t^{\prime}} \in \mathcal{O}_{k}^{\times} .
$$

Note that $c_{4}(E) \neq 0$ since $j(E) \neq 0$.

Consequently, to investigate elliptic curves having everywhere good reduction over a real quadratic field $k$ with unit discriminant and admitting a 3-isogeny defined over $k$, we need to study the equations

$$
X^{3}=u+27 v, \quad X^{3}=u+v, \quad X^{3}=\pi^{3} u+\pi^{\prime 3} v
$$

in $X \in \mathcal{O}_{k} \backslash\{0\}, u, v \in \mathcal{O}_{k}^{\times}$, where $\pi \in \mathcal{O}_{k}, N_{k / \mathbb{Q}}(\pi)= \pm 3$. We will study them in the next subsection.

\subsection{Some Diophantine equations}

Lemma 4. Let $k$ be a quadratic field with $\left(h_{k}, 6\right)=1$. Then the equation

$$
X^{3}=1+27 u, \quad X \in \mathcal{O}_{k}, u \in \mathcal{O}_{k}^{\times},
$$

has a solution only when $k=\mathbb{Q}(\sqrt{6})$ or $\mathbb{Q}(\sqrt{33})$, in which cases, the only solutions are $(X, u)=(4 \pm \sqrt{6}, 5 \pm 2 \sqrt{6}),(-(5 \pm \sqrt{33}),-(23 \pm 4 \sqrt{33}))$, respectively. Note that $h_{\mathbb{Q}(\sqrt{6})}=h_{\mathbb{Q}(\sqrt{33})}=1$, and that $5+2 \sqrt{6}$ (resp. $23+4 \sqrt{33})$ is the fundamental unit of $\mathbb{Q}(\sqrt{6})$ (resp. $\mathbb{Q}(\sqrt{33}))$.

Proof. First consider the case where 3 is ramified. Since $h_{k}$ is odd, we have $(3)=\left(\pi^{2}\right), \pi \in \mathcal{O}_{k}$. By (3.8) we have

$$
X-1=\pi^{a} v, \quad X^{2}+X+1=\pi^{6-a} w, \quad v, w \in \mathcal{O}_{k}^{\times}, a \in \mathbb{Z}, 0 \leq a \leq 6,
$$

whence

$$
\pi^{2 a} v^{2}+3 \pi^{a} v+3=\pi^{6-a} w .
$$

If $a=0,2,3,5$ or 6 , then the $\pi$-adic values of both sides of (3.9) cannot be equal. If $a=1$, then taking the norm of both sides of (3.9) yields

$$
\operatorname{Tr}_{k / \mathbb{Q}}(\pi v)^{2}+\left(N_{k / \mathbb{Q}}(\pi v)+3\right) \operatorname{Tr}_{k / \mathbb{Q}}(\pi v)+\left(N_{k / \mathbb{Q}}(\pi v)+6\right)= \pm 3^{4} .
$$

If $N_{k / \mathbb{Q}}(\pi v)=-3$, then $\operatorname{Tr}_{k / \mathbb{Q}}(\pi v)$ cannot be rational. If $N_{k / \mathbb{Q}}(\pi v)=3$, then $\operatorname{Tr}_{k / \mathbb{Q}}(\pi v)=-12$ or 6 . The former corresponds to $(X, u)=(-(5 \pm \sqrt{33})$, $-(23 \pm 4 \sqrt{33}))$, the latter to $(X, u)=(4 \pm \sqrt{6}, 5 \pm 2 \sqrt{6})$. If $a=4$, then we similarly obtain

$$
\operatorname{Tr}_{k / \mathbb{Q}}\left(\pi^{4} v\right)^{2}+\left(N_{k / \mathbb{Q}}\left(\pi^{4} v\right)+3\right) \operatorname{Tr}_{k / \mathbb{Q}}\left(\pi^{4} v\right)+\left(N_{k / \mathbb{Q}}\left(\pi^{4} v\right)+3+3^{7}\right)= \pm 3 .
$$

For all possibilities of the values of $N_{k / \mathbb{Q}}\left(\pi^{4} v\right)$ and the signs of the right hand side, $\operatorname{Tr}_{k / \mathbb{Q}}\left(\pi^{4} v\right)$ cannot be rational.

If 3 is inert, a similar argument works and we can show that there are no solutions in this case. 
Finally, consider the case where $(3)=\mathfrak{p p}^{\prime}, \mathfrak{p} \neq \mathfrak{p}^{\prime}$. Then, for some $a, a^{\prime} \in \mathbb{Z}, 0 \leq a, a^{\prime} \leq 3$, we have

$$
(X-1)=\mathfrak{p}^{a} \mathfrak{p}^{\prime a^{\prime}}, \quad\left(X^{2}+X+1\right)=\mathfrak{p}^{3-a} \mathfrak{p}^{\prime 3-a^{\prime}} .
$$

If $a=a^{\prime}$, then we have $(X-1)=(3)^{a},\left(X^{2}+X+1\right)=(3)^{3-a}$. Hence a similar argument works and we can show that there are no solutions in this case. Suppose that $a \neq a^{\prime}$. Considering the conjugate of (3.8) if necessary, we may assume that $a<a^{\prime}$. Then $(X-1)=\left(\mathfrak{p p}^{\prime}\right)^{a} \mathfrak{p}^{\prime a^{\prime}-a}=(3)^{a} \mathfrak{p}^{\prime a^{\prime}-a}$, and $a^{\prime}-a=1,2,3$. Since $\left(h_{k}, 6\right)=1$, it follows that $\mathfrak{p}$ and $\mathfrak{p}^{\prime}$ are both principal. Thus a similar argument leads to the conclusion that there are no solutions also in this case.

We can prove the following three lemmas similarly.

Lemma 5 (Kida [6]). Let $k$ be a quadratic field. Then the equation

$$
X^{3}=u+27, \quad X \in \mathcal{O}_{k}, u \in \mathcal{O}_{k}^{\times},
$$

has no solutions.

Lemma 6. Let $k$ be a quadratic field. Then the only solution of the equation

$$
X^{3}=1+u, \quad X \in \mathcal{O}_{k}, u \in \mathcal{O}_{k}^{\times},
$$

is $(X, u)=(0,-1)$.

Lemma 7. Let $k$ be a real quadratic field in which 3 splits into two principal prime ideals with generators $\pi$ and $\pi^{\prime}$. Then the equation

$$
X^{3}=\pi^{3}+\pi^{\prime 3} u, \quad X \in \mathcal{O}_{k}, u \in \mathcal{O}_{k}^{\times},
$$

has no solutions.

We give two more results on these equations.

LEMMA 8. If the norm of the fundamental unit of a real quadratic field $k$ is 1 and

$$
X^{3}=u-v, \quad X \in \mathcal{O}_{k}, u, v \in \mathcal{O}_{k}^{\times}, u v \in \mathcal{O}_{k}^{\times 2},
$$

then $X=0$.

Pr o of. By assumption, we have $u v^{\prime}=w^{2}$ for some $w \in \mathcal{O}_{k}^{\times}$. Taking the norm of both sides of (3.10) and noting $N_{k / \mathbb{Q}}(u)=N_{k / \mathbb{Q}}(v)=N_{k / \mathbb{Q}}(w)=1$, we obtain

$$
\operatorname{Tr}_{k / \mathbb{Q}}(w)^{2}=\left\{-N_{k / \mathbb{Q}}(X)\right\}^{3}+4 .
$$

It then follows that $X=0$, since the only (affine) $\mathbb{Q}$-rational points of the elliptic curve $y^{2}=x^{3}+4$, which is the curve 108A1 in Table 1 of [2], are $(0, \pm 2)$. 
Lemma 9. Let $k$ be one of the real quadratic fields $\mathbb{Q}(\sqrt{33}), \mathbb{Q}(\sqrt{57})$, $\mathbb{Q}(\sqrt{69})$ and $\mathbb{Q}(\sqrt{93})$. Then the equation

$$
X^{3}=u+27 v, \quad X \in \mathcal{O}_{k}, u, v \in \mathcal{O}_{k}^{\times}, u \notin k^{\times 3},
$$

has no solutions.

Pr o of. Equation (3.11) has no solutions modulo 3, 3, 9 or $(31+3 \sqrt{93}) / 2$ according as $k=\mathbb{Q}(\sqrt{33}), \mathbb{Q}(\sqrt{57}), \mathbb{Q}(\sqrt{69})$ or $\mathbb{Q}(\sqrt{93})$. Note that

$$
\mathcal{O}_{\mathbb{Q}(\sqrt{93})} /((31+3 \sqrt{93}) / 2) \cong \mathbb{Z} / 31 \mathbb{Z}
$$

3.3. Subgroups of $\mathrm{GL}_{2}\left(\mathbb{F}_{3}\right)$ as a Galois group. Let $k$ be an algebraic number field not containing $\sqrt{-3}$. Let $E$ be an elliptic curve defined over $k$, let $E[3]=\{P \in E \mid 3 P=O\}$ be the group of 3-division points of $E$, and let $L=k(E[3])$ be the field generated over $k$ by the points of $E[3]$. We may regard $G=\operatorname{Gal}(L / k)$ as a subgroup of $\mathrm{GL}_{2}\left(\mathbb{F}_{3}\right)$ by the faithful representation $G \rightarrow \mathrm{GL}_{2}\left(\mathbb{F}_{3}\right)$ induced by the action of $G$ on $E[3]$. Here we study what group $G$ can be. We mention that Naito [10] studied the same problem for elliptic curves defined over $\mathbb{Q}$.

Lemma 10. Let $G$ be as above. Let $\varrho=\left(\begin{array}{cc}1 & 0 \\ 0 & -1\end{array}\right), \sigma=\left(\begin{array}{ll}0 & 1 \\ 1 & 0\end{array}\right), \tau=\left(\begin{array}{ll}1 & -1 \\ 1 & 1\end{array}\right) \in$ $\mathrm{GL}_{2}\left(\mathbb{F}_{3}\right)$, which satisfy the relations $\varrho^{2}=\sigma^{2}=\tau^{8}=1, \sigma \tau \sigma^{-1}=\tau^{3}$. Then

(1) $G$ is conjugate in $\mathrm{GL}_{2}\left(\mathbb{F}_{3}\right)$ to one of the following:

(i) $\langle\varrho\rangle \cong \mathbb{Z} / 2 \mathbb{Z}$.

(ii) $\langle-1\rangle \times\langle\varrho\rangle \cong \mathbb{Z} / 2 \mathbb{Z} \times \mathbb{Z} / 2 \mathbb{Z}$.

(iii) $\left(\begin{array}{c}1 * \\ 0 *\end{array}\right) \cong S_{3}$ (the symmetric group of degree 3 ).

(iv) $\left(\begin{array}{c}* * \\ 0\end{array}\right) \cong S_{3}$.

(v) $\left\langle\sigma, \tau^{2}\right\rangle \cong D_{8}$ (the dihedral group of order 8 ).

(vi) $\langle\tau\rangle \cong \mathbb{Z} / 8 \mathbb{Z}$.

(vii) $\left(\begin{array}{l}* * \\ 0 *\end{array}\right) \cong S_{3} \times \mathbb{Z} / 2 \mathbb{Z}$.

(viii) $\langle\sigma, \tau\rangle \cong S D_{16}$ (the semi-dihedral group of order 16 ).

(ix) $\mathrm{GL}_{2}\left(\mathbb{F}_{3}\right)$.

(2) $\Delta(E)$ is a cube in $k$ if and only if $G$ is conjugate in $\mathrm{GL}_{2}\left(\mathbb{F}_{3}\right)$ to one of the groups in (i), (ii), (v), (vi) or (viii). For each case, $G \cap \mathrm{SL}_{2}\left(\mathbb{F}_{3}\right)=$ $\operatorname{Gal}(L / k(\sqrt{-3}))$ is conjugate in $\mathrm{GL}_{2}\left(\mathbb{F}_{3}\right)$ to $\{1\},\langle-1\rangle \cong \mathbb{Z} / 2 \mathbb{Z},\left\langle\tau^{2}\right\rangle \cong \mathbb{Z} / 4 \mathbb{Z}$, $\left\langle\tau^{2}\right\rangle \cong \mathbb{Z} / 4 \mathbb{Z},\left\langle\sigma \tau, \tau^{2}\right\rangle \cong Q_{8}$ (the quaternion group), respectively.

(3) E admits a 3-isogeny defined over $k$ if and only if $G$ is conjugate in $\mathrm{GL}_{2}\left(\mathbb{F}_{3}\right)$ to one of the groups in (i), (ii), (iii), (iv) or (vii).

REMARK. $\mathrm{GL}_{2}\left(\mathbb{F}_{3}\right)$ is of order $2^{4} \cdot 3$ and hence $S D_{16}$ is a 2-Sylow subgroup of $\mathrm{GL}_{2}\left(\mathbb{F}_{3}\right)$. 
Proof (of Lemma 10). (1) We have $\# G \geq 2$, since $k(\sqrt{-3}) \subset L$ ([17], p. 98) and $[k(\sqrt{-3}): k]=2$. The special linear group $\mathrm{SL}_{2}\left(\mathbb{F}_{3}\right)$ does not contain $G$, since we have $\operatorname{Gal}(L / k(\sqrt{-3}))=G \cap \mathrm{SL}_{2}\left(\mathbb{F}_{3}\right)$ by the commutativity of the diagram

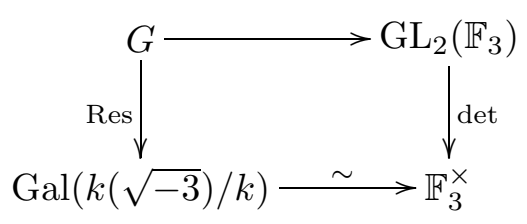

From these together with the classification of the subgroups of $\mathrm{GL}_{2}\left(\mathbb{F}_{3}\right)$ (cf. [10]), we obtain the assertion.

(2) The first part is clear from the fact that $\Delta(E)$ is a cube in $k$ if and only if $[L: k]$ is not divisible by $3([14], \S 5.3)$. The second part follows from direct calculation.

(3) Since admitting a 3-isogeny defined over $k$ is equivalent to the existence of a point $P$ of order 3 such that $\sigma(P)= \pm P$ for any $\sigma \in G$, we may assume, by an appropriate choice of a basis of $E[3]$, that $G$ is a subgroup of $\left(\begin{array}{l}* * \\ 0 *\end{array}\right)$. The groups appearing in (1) which are subgroups of this group are those in (i), (ii), (iii), (iv) and (vii).

4. Some criteria. In this section, we use the following notation: For subgroups $H$ and $N$ of $\mathrm{GL}_{2}\left(\mathbb{F}_{3}\right), H \sim N$ means that $H$ is conjugate in $\mathrm{GL}_{2}\left(\mathbb{F}_{3}\right)$ to $N$.

Proposition 11. Let $k$ be a real quadratic field. Assume $h_{k}\left((3) \mathfrak{p}_{\infty}^{(1)} \mathfrak{p}_{\infty}^{(2)}\right)$ $\not \equiv 0(\bmod 4)$, where $\mathfrak{p}_{\infty}^{(1)}$ and $\mathfrak{p}_{\infty}^{(2)}$ are the real primes of $k$, or $h_{k(\sqrt{-3})}((3)) \not \equiv$ $0(\bmod 4)$. Then every elliptic curve $E$ with everywhere good reduction over $k$ whose discriminant $\Delta(E)$ is a cube in $k$ admits a 3-isogeny defined over $k$.

Proof. Let $E$ be an elliptic curve with everywhere good reduction over $k$ with $\Delta(E) \in k^{\times 3}$. Set $L:=k(E[3]), G:=\operatorname{Gal}(L / k)$ and $H:=$ $\operatorname{Gal}(L / k(\sqrt{-3}))=G \cap \mathrm{SL}_{2}\left(\mathbb{F}_{3}\right)$. By Lemma $10(2), G$ is conjugate in $\mathrm{GL}_{2}\left(\mathbb{F}_{3}\right)$ to $\langle\sigma, \tau\rangle \cong S D_{16},\langle\tau\rangle \cong \mathbb{Z} / 8 \mathbb{Z},\left\langle\sigma, \tau^{2}\right\rangle \cong D_{8},\langle-1\rangle \times\langle\varrho\rangle \cong \mathbb{Z} / 2 \mathbb{Z} \times \mathbb{Z} / 2 \mathbb{Z}$, or $\langle\varrho\rangle \cong \mathbb{Z} / 2 \mathbb{Z}$. If $G \sim\langle\tau\rangle$ or $\left\langle\sigma, \tau^{2}\right\rangle$, then it is clear that $G$ has a normal subgroup $N$ such that $G / N$ is of order 4 . Further, by Lemma 10(2), $H \cong \mathbb{Z} / 4 \mathbb{Z}$ in these cases. If $G \sim\langle\sigma, \tau\rangle$, then $G$ has a normal subgroup of $N$ with $G / N \cong \mathbb{Z} / 2 \mathbb{Z} \times \mathbb{Z} / 2 \mathbb{Z}$. Indeed, $\langle\sigma, \tau\rangle /\left\langle\tau^{2}\right\rangle \cong \mathbb{Z} / 2 \mathbb{Z} \times \mathbb{Z} / 2 \mathbb{Z}$. Further $H \sim\left\langle\sigma \tau, \tau^{2}\right\rangle \cong Q_{8}$ and $\left\langle\sigma \tau, \tau^{2}\right\rangle /\left\langle\tau^{2}\right\rangle \cong \mathbb{Z} / 2 \mathbb{Z} \times \mathbb{Z} / 2 \mathbb{Z}$. Thus in view of the criterion of Néron-Ogg-Shafarevich ([17], p. 184), our assumptions on ray class numbers imply that $G \sim\langle\varrho\rangle$ or $\langle-1\rangle \times\langle\varrho\rangle$. We therefore see from Lemma $10(3)$ that $E$ admits a 3 -isogeny defined over $k$. 
Proposition 12. Let $k$ be a real quadratic field with $\left(h_{k}, 6\right)=1$. Let $\varepsilon$ be the fundamental unit of $k$ and let $\mathfrak{P}_{\infty}^{(1)}$ and $\mathfrak{P}_{\infty}^{(2)}$ be the real primes of $k(\sqrt[3]{\varepsilon})$.

(1) If $h_{k(\sqrt[3]{\varepsilon})}\left((3) \mathfrak{P}_{\infty}^{(1)} \mathfrak{P}_{\infty}^{(2)}\right) \not \equiv 0(\bmod 4)$ or $h_{k(\sqrt[3]{\varepsilon}, \sqrt{-3})}((3)) \not \equiv 0(\bmod 4)$, then every elliptic curve $E$ with everywhere good reduction over $k$ whose discriminant $\Delta(E)$ is not a cube in $k$ admits a 3-isogeny defined over $k$.

(2) If $h_{k(\sqrt[3]{\varepsilon})}\left((3) \mathfrak{P}_{\infty}^{(1)} \mathfrak{P}_{\infty}^{(2)}\right) \not \equiv 0(\bmod 4)$ or $h_{k(\sqrt[3]{\varepsilon}, \sqrt{-3})}((3)) \not \equiv 0(\bmod 2)$, then every elliptic curve $E$ with everywhere good reduction over $k$ whose discriminant $\Delta(E)$ is not a cube in $k$ has a $k$-rational subgroup $V$ of order 3 , and either $E$ or $E / V$ has a k-rational point of order 3 .

Proof. (1) Let $E$ be an elliptic curve with everywhere good reduction over $k$ and let $L=k(E[3]), G=\operatorname{Gal}(L / k)$. By the corollary to Theorem 1 of [15], which states that every elliptic curve with everywhere good reduction over $k$ has a global minimal model provided $\left(h_{k}, 6\right)=1$, and the assumption that $\Delta(E)$ is not a cube, we have $k(\sqrt[3]{\Delta(E)})=k(\sqrt[3]{\varepsilon})$. Since $L$ contains $k(\sqrt[3]{\Delta(E)})$ ([14], p. 305), we have $[L: k] \equiv 0(\bmod 3)$. Thus, by Lemma $10(2)$, we have $G \sim\left(\begin{array}{c}1 * \\ 0 *\end{array}\right),\left(\begin{array}{c}* * \\ 0\end{array}\right),\left(\begin{array}{c}* * \\ 0 *\end{array}\right)$ or $\mathrm{GL}_{2}\left(\mathbb{F}_{3}\right)$. Suppose that $E$ admits no 3 -isogeny defined over $k$. Then, by Lemma 10(3), we have $G=\mathrm{GL}_{2}\left(\mathbb{F}_{3}\right), \operatorname{Gal}(L / k(\sqrt[3]{\varepsilon})) \sim\langle\sigma, \tau\rangle$ and $\operatorname{Gal}(L / k(\sqrt[3]{\varepsilon}, \sqrt{-3}))=$ $\operatorname{Gal}(L / k(\sqrt[3]{\varepsilon})) \cap \mathrm{SL}_{2}\left(\mathbb{F}_{3}\right) \sim\left\langle\sigma \tau, \tau^{2}\right\rangle$. The criterion of Néron-Ogg-Shafarevich and the fact that $\langle\sigma, \tau\rangle /\left\langle\tau^{2}\right\rangle$ and $\left\langle\sigma \tau, \tau^{2}\right\rangle /\left\langle\tau^{2}\right\rangle$ are both isomorphic to $\mathbb{Z} / 2 \mathbb{Z} \times$ $\mathbb{Z} / 2 \mathbb{Z}$ imply $h_{k(\sqrt[3]{\varepsilon})}\left((3) \mathfrak{P}_{\infty}^{(1)} \mathfrak{P}_{\infty}^{(2)}\right) \equiv 0(\bmod 4)$ and $h_{k(\sqrt[3]{\varepsilon}, \sqrt{-3})}((3)) \equiv 0$ $(\bmod 4)$.

(2) According to (1), we have $G \sim\left(\begin{array}{l}1 * \\ 0 *\end{array}\right),\left(\begin{array}{l}* * \\ 01\end{array}\right)$ or $\left(\begin{array}{l}* * \\ 0 *\end{array}\right)$. Supposing $G \sim\left(\begin{array}{c}* * \\ 0 *\end{array}\right)$, the criterion of Néron-Ogg-Shafarevich implies that $L / k(\sqrt[3]{\varepsilon})$ is an abelian extension of degree 4 unramified outside $\left\{3, \mathfrak{P}_{\infty}^{(1)}, \mathfrak{P}_{\infty}^{(2)}\right\}$ and $L / k(\sqrt[3]{\varepsilon}, \sqrt{-3})$ is a quadratic extension unramified outside 3 . These contradict our assumptions.

5. Proof of Theorems 1 and 2. Let $k$ be one of the real quadratic fields $\mathbb{Q}(\sqrt{33}), \mathbb{Q}(\sqrt{57}), \mathbb{Q}(\sqrt{69})$ and $\mathbb{Q}(\sqrt{93})$. The fundamental unit of $k$ is

$$
\varepsilon= \begin{cases}23+4 \sqrt{33} & \text { if } k=\mathbb{Q}(\sqrt{33}), \\ 151+20 \sqrt{57} & \text { if } k=\mathbb{Q}(\sqrt{57}), \\ (25+3 \sqrt{69}) / 2 & \text { if } k=\mathbb{Q}(\sqrt{69}), \\ (29+3 \sqrt{93}) / 2 & \text { if } k=\mathbb{Q}(\sqrt{93}) .\end{cases}
$$

Note that $N_{k / \mathbb{Q}}(\varepsilon)=1$. Let $E$ be an elliptic curve with everywhere good reduction over $k$. Table 1 and Propositions 11 and 12 imply that $E$ admits a 3-isogeny defined over $k$. 
Table 1. Ray class numbers

\begin{tabular}{ccccc}
\hline$k$ & $h_{k}\left((3) \mathfrak{p}_{\infty}^{(1)} \mathfrak{p}_{\infty}^{(2)}\right)$ & $h_{k(\sqrt{-3})}((3))$ & $h_{k(\sqrt[3]{\varepsilon})}\left((3) \mathfrak{P}_{\infty}^{(1)} \mathfrak{P}_{\infty}^{(2)}\right)$ & $h_{k(\sqrt[3]{\varepsilon}, \sqrt{-3})}((3))$ \\
\hline $\mathbb{Q}(\sqrt{33})$ & 2 & 1 & $2 \cdot 3^{3}$ & $3^{5}$ \\
$\mathbb{Q}(\sqrt{57})$ & $\mathbf{4}$ & 2 & $\mathbf{2}^{\mathbf{2}} \cdot \mathbf{3}$ & $2 \cdot 3^{3}$ \\
$\mathbb{Q}(\sqrt{69})$ & 6 & 9 & $2 \cdot 3$ & $3^{2}$ \\
$\mathbb{Q}(\sqrt{93})$ & $\mathbf{1 2}$ & 18 & $\mathbf{2}^{\mathbf{2}} \cdot \mathbf{3}$ & $2 \cdot 3^{2}$ \\
\hline
\end{tabular}

In the case where $\Delta(E)$ is a cube in $k, k$ is $\mathbb{Q}(\sqrt{33})$ and $E$ is isomorphic over $k$ to $E_{1}$ or $E_{1}^{\prime}$. More generally we have the following:

Proposition 13. Let $k$ be a quadratic field with $\left(h_{k}, 6\right)=1$. If there is an elliptic curve $E$ which has everywhere good reduction over $k$ and admits a 3-isogeny defined over $k$, and whose discriminant $\Delta(E)$ is a cube in $k$, then $k$ is equal to $\mathbb{Q}(\sqrt{6})$ or $\mathbb{Q}(\sqrt{33})$. If $k=\mathbb{Q}(\sqrt{6})$ (resp. $k=\mathbb{Q}(\sqrt{33})$ ), then such a curve $E$ is isomorphic over $k$ to

$E_{4}: y^{2}+(4+\sqrt{6}) x y+(5+2 \sqrt{6}) y=x^{3}, \Delta\left(E_{4}\right)=(5+2 \sqrt{6})^{3}, j\left(E_{4}\right)=8000$ or $E_{4}^{\prime}$ (resp. to $E_{1}$ or $\left.E_{1}^{\prime}\right)$.

For the proof of this lemma and for later use, we first give a lemma.

Lemma 14 (Kida [8]). Let $E$ be an elliptic curve having everywhere good reduction over a quadratic field $k$. Let $s$ denote the number of ramifying rational primes in the extension $k / \mathbb{Q}$. Then the number of twists of $E$ having everywhere good reduction over $k$ is $2^{s-1}$.

Pr o of (of Proposition 13). By the argument in Subsection 3.1, $j(E)$ is of the form $J(t), t \in \mathcal{O}_{k}, t \mid 3^{6}$, and the principal ideal $(t)$ is a sixth power. By (3.3)-(3.7), we see that there exist $X \in \mathcal{O}_{k} \backslash\{0\}$ and $u \in \mathcal{O}_{k}^{\times}$such that

$$
\begin{array}{ll}
X^{3}=1+27 u & \text { if }(t)=(1), \\
X^{3}=u+27 & \text { if }(t)=(729), \\
X^{3}=1+u & \text { if } 3 \text { is ramified, and }(t)=(27), \\
X^{3}=\pi^{3}+\pi^{\prime 3} u & \text { if } 3= \pm \pi \pi^{\prime}, \text { and }(t)=\left(\pi^{6}\right) \text { or }\left(\pi^{\prime 6}\right) .
\end{array}
$$

Note that in equation (5.1), $u=1 / t, X=c_{4}(E) /((t+3) v)$, where $\Delta(E)=$ $v^{3}, v \in \mathcal{O}_{k} \backslash\{0\}$. From Lemmas $5-7$, none of the equations (5.2)-(5.4) has solutions. From Lemma 4 , the only units $u$ satisfying equation (5.1) are $u=5 \pm 2 \sqrt{6}$ and $-(23 \pm 4 \sqrt{33})$. If $u=5 \pm 2 \sqrt{6}$ (resp. $u=-(23 \pm 4 \sqrt{33})$ ), then $j(E)=J(5 \mp 2 \sqrt{6})=8000($ resp. $j(E)=J(-(23 \mp 4 \sqrt{33}))=-32768)$. We have two elliptic curves with everywhere good reduction over $\mathbb{Q}(\sqrt{6})$ (resp. $\mathbb{Q}(\sqrt{33})$ ) with $j$ invariant 8000 (resp. -32768 ), namely $E_{4}$ and $E_{4}^{\prime}$ (resp. $E_{1}$ and $\left.E_{1}^{\prime}\right)$. Lemma 14 therefore implies our assertion. 
REMARK. All elliptic curves with everywhere good reduction over $\mathbb{Q}(\sqrt{6})$ have been determined in [5], [7].

Consider the case where $\Delta(E)$ is not a cube in $k$. The field $K:=$ $k(\sqrt{\Delta(E)})$ is one of the fields $k, k(\sqrt{-1})$ or $k(\sqrt{ \pm \varepsilon})$, since we may assume that $\Delta(E)$ is a unit (see the above-cited result in [15]). The field generated over $k$ by the points of order 2 of $E$ is a cyclic cubic extension of $K$, since in [1], it is shown that $E$ has no $k$-rational points of order 2 . This means that, in view of the criterion of Néron-Ogg-Shafarevich, $h_{K}^{(2)}:=h_{K}\left(\prod_{\mathfrak{p} \mid 2} \mathfrak{p}\right)$ is divisible by 3 . Thus Table 2 implies that $\Delta(E)=-\varepsilon^{2 n+1}(n \in \mathbb{Z})$.

Table 2. $h_{K}^{(2)}(K=k, k(\sqrt{-1}), k(\sqrt{ \pm \varepsilon}))$

\begin{tabular}{ccccc}
\hline \multirow{2}{*}{$k$} & \multicolumn{4}{c}{$h_{K}^{(2)}$} \\
\cline { 2 - 5 } & $K=k$ & $K=k(\sqrt{-1})$ & $K=k(\sqrt{\varepsilon})$ & $K=k(\sqrt{-\varepsilon})$ \\
\hline $\mathbb{Q}(\sqrt{33})$ & 1 & 2 & 1 & $\mathbf{3}$ \\
$\mathbb{Q}(\sqrt{57})$ & 1 & 2 & 1 & $\mathbf{3}$ \\
$\mathbb{Q}(\sqrt{69})$ & 1 & 4 & 1 & $\mathbf{3}$ \\
$\mathbb{Q}(\sqrt{93})$ & 1 & 2 & 1 & $\mathbf{3}$ \\
\hline
\end{tabular}

In view of the formulae for an admissible change of variables, we may assume that $\Delta(E)=-\varepsilon^{ \pm 1}$ or $-\varepsilon^{ \pm 5}$. We may further assume that $\Delta(E)=$ $-\varepsilon^{6 n+1}(n=0,-1)$ by considering the conjugate of $E$.

Suppose first that $(t)=(1)$. By (3.3), we obtain

$$
X^{3}=\varepsilon+27 u, \quad X=\frac{-c_{4}(E)}{(t+3) \varepsilon^{2 n}} \in \mathcal{O}_{k}, \quad u=\frac{\varepsilon}{t} \in \mathcal{O}_{k}^{\times},
$$

which is impossible by Lemma 9 .

Suppose next that $(t)=(27)$. Then, by (3.5), we obtain

$$
X^{3}=\varepsilon+\varepsilon u, \quad X=\frac{-c_{4}(E)}{(t+3) \varepsilon^{2 n}} \in \mathcal{O}_{k} \backslash\{0\}, \quad u=\frac{27}{t} \in \mathcal{O}_{k}^{\times} .
$$

Let

$$
\pi= \begin{cases}6+\sqrt{33} & \text { if } k=\mathbb{Q}(\sqrt{33}), \\ 15+2 \sqrt{57} & \text { if } k=\mathbb{Q}(\sqrt{57}), \\ (9+\sqrt{69}) / 2 & \text { if } k=\mathbb{Q}(\sqrt{69}), \\ (9+\sqrt{93}) / 2 & \text { if } k=\mathbb{Q}(\sqrt{93})\end{cases}
$$

be a prime element of $k$ dividing 3. Lemma 3(1) and the fact that $\pi^{2}=3 \varepsilon$ imply $u=-\varepsilon^{2 m}, m \in \mathbb{Z}$, whence

$$
X^{3}=\varepsilon-\varepsilon^{2 m+1}, \quad X \neq 0,
$$

which is impossible by Lemma 8 . 
Finally, suppose that $(t)=(729)$. Since $t / \Delta(E)=-t / \varepsilon^{6 n+1}$ is a square by Lemma $3(1)$, we have $729 / t=-\varepsilon^{2 m-1}$ for some $m \in \mathbb{Z}$, and hence by (3.4) we have

$$
\left(\frac{3 c_{4}(E)}{(t+3) \varepsilon^{2 n}}\right)^{3}=\varepsilon^{2 m}-27 \varepsilon .
$$

By Lemma 9, $m$ must be a multiple of 3 . Letting $l=m / 3 \in \mathbb{Z}$, we have

$$
X^{3}=1-27 \varepsilon^{1-6 l}, \quad X=\frac{3 c_{4}(E)}{(t+3) \varepsilon^{2 n+2 l}} \in \mathcal{O}_{k} .
$$

By Lemma 4 , this is possible only if $k=\mathbb{Q}(\sqrt{33}), l=0$, whence $j(E)=$ $J(-729 \varepsilon)=-(5+\sqrt{33})^{3}(5588+972 \sqrt{33})^{3} \varepsilon^{-1}$, which equals $j\left(E_{2}\right)$ and $j\left(E_{3}^{\prime}\right)$. Lemma 14 therefore implies that $E$ is isomorphic over $\mathbb{Q}(\sqrt{33})$ to $E_{2}$ or $E_{3}^{\prime}$ according as $\Delta(E)=-\varepsilon$ or $\Delta(E)=-\varepsilon^{-5}$.

The proof is now complete.

6. Appendix. In Section 5, we gave a characterization of elliptic curves having everywhere good reduction over a real quadratic field $k$, admitting a 3-isogeny defined over $k$, and having cubic discriminant (Proposition 13). Here we give a similar characterization of the curves whose discriminant is equal to $\square$, a square in $k$, or $-\square$. More precisely, we prove

Proposition 15. Let $k$ be a real quadratic field. If there exists an elliptic curve $E$ with everywhere good reduction over $k$ given by a global minimal model with $j(E)=J(t)\left(t \in \mathcal{O}_{k},(t)=(1)\right.$ or $\left.(729)\right)$ and $\Delta(E)= \pm \square$, then $k=\mathbb{Q}(\sqrt{29})$ and $E$ is isomorphic over $k$ to

$$
\begin{aligned}
E_{5}: & y^{2}+x y+\varepsilon^{2} y=x^{3}, \\
& \Delta\left(E_{5}\right)=-\varepsilon^{10}, \quad j\left(E_{5}\right)=\left(\varepsilon^{2}-3\right)^{3} / \varepsilon^{4}, \\
E_{6}: & y^{2}+x y+\varepsilon^{2} y=x^{3}-5 \varepsilon^{2} x-\left(\varepsilon^{2}+7 \varepsilon^{4}\right), \\
& \Delta\left(E_{6}\right)=-\varepsilon^{14}, \quad j\left(E_{6}\right)=-\left(1+216 \varepsilon^{2}\right)^{3} / \varepsilon^{14},
\end{aligned}
$$

or to their conjugates $E_{5}^{\prime}, E_{6}^{\prime}$. Here $\varepsilon=(5+\sqrt{29}) / 2$ is the fundamental unit of $\mathbb{Q}(\sqrt{29})$ and $J$ is the one given in Subsection 3.1 .

Lemma 16. (1) The equation $27 Y^{2}=X^{3}-676(X, Y \in \mathbb{Z})$ has no solutions.

(2) The equation $27 Y^{2}=X^{3}+784(X, Y \in \mathbb{Z})$ has no solutions.

(3) The only $X, Y \in \mathbb{Z}$ satisfying $27 Y^{2}=X^{3}+676$ are $(X, Y)=$ $(-1, \pm 5),(26, \pm 26)$.

(4) The only $X, Y \in \mathbb{Z}$ satisfying $27 Y^{2}=X^{3}-784$ are $(X, Y)=$ $(19, \pm 15),(28, \pm 28)$. 
Pr o of. Let $A$ be one of $\pm 676, \pm 784$. If $X, Y \in \mathbb{Z}$ satisfy $27 Y^{2}=X^{3}+A$, then $(3 X, 27 Y)$ is an integral point of the elliptic curve

$$
C_{A}: y^{2}=x^{3}+27 A \text {. }
$$

If $A=784$ or -676 , then $C_{A}(\mathbb{Q})=\{O\}$ is shown by 2-descent. The only integral points on $C_{676}$ are

$$
(-26, \pm 26),(-3, \pm 135),(13, \pm 143),(22, \pm 170),(78, \pm 702),(1573, \pm 62387),
$$

among which $(-3, \pm 135)=(3 \cdot(-1), \pm 27 \cdot 5)$ and $(78, \pm 702)=(3 \cdot 26, \pm 27 \cdot 26)$ provide solutions of $27 Y^{2}=X^{3}+676$. The only integral points on $C_{-784}$ are

$$
(28, \pm 28),(57, \pm 405),(84, \pm 756),(1708, \pm 70588),
$$

among which $(57, \pm 405)=(3 \cdot 19, \pm 27 \cdot 15)$ and $(84, \pm 756)=(3 \cdot 28, \pm 27 \cdot 28)$ provide solutions of $27 Y^{2}=X^{3}-784$. (The computations of the integral points of $C_{676}$ and $C_{-784}$ are done using KASH version 2.1.)

LEmma 17. Let $k$ be a real quadratic field. If there exist $u, v \in \mathcal{O}_{k}^{\times}$, $X \in \mathcal{O}_{k}$ such that

$$
X^{3}=u+27 v, \quad u v= \pm \square,
$$

then $k$ is equal to $\mathbb{Q}(\sqrt{29})$ and the only solutions are $(u, v, X)=\left( \pm \varepsilon^{3 n+1}\right.$, $\left.\mp \varepsilon^{3 n-1}, \mp \varepsilon^{n-1}\right),\left( \pm \varepsilon^{3 n-1}, \mp \varepsilon^{3 n+1}, \mp \varepsilon^{n+1}\right)(n \in \mathbb{Z})$, where $\varepsilon=(5+\sqrt{29}) / 2$ is the fundamental unit of $\mathbb{Q}(\sqrt{29})$.

Proof. By changing $(u, v, X)$ to $\left(u^{4}, u^{3} v, u X\right)$ if necessary, we may assume that $N_{k / \mathbb{Q}}(u)=N_{k / \mathbb{Q}}(v)=1$. Taking the norm of both sides of (6.1), we have $N_{k / \mathbb{Q}}(X)^{3}=730+27 \operatorname{Tr}_{k / \mathbb{Q}}\left(u v^{\prime}\right)$. Since $u v= \pm \square$ and $N_{k / \mathbb{Q}}(v)=1$, we have $u v^{\prime}=u v / v^{2}= \pm w^{2}$ for some $w \in \mathcal{O}_{k}^{\times}$. Hence

$$
N_{k / \mathbb{Q}}(X)^{3}=730 \pm 27 \operatorname{Tr}_{k / \mathbb{Q}}\left(w^{2}\right)=730 \pm 27\left\{\operatorname{Tr}_{k / \mathbb{Q}}(w)^{2}-2 N_{k / \mathbb{Q}}(w)\right\} .
$$

If the sign is + , then

$$
\begin{aligned}
27 \operatorname{Tr}_{k / \mathbb{Q}}(w)^{2} & =N_{k / \mathbb{Q}}(X)^{3}-730+54 N_{k / \mathbb{Q}}(w) \\
& = \begin{cases}N_{k / \mathbb{Q}}(X)^{3}-676 & \text { if } N_{k / \mathbb{Q}}(w)=1, \\
N_{k / \mathbb{Q}}(X)^{3}-784 & \text { if } N_{k / \mathbb{Q}}(w)=-1 .\end{cases}
\end{aligned}
$$

It follows from Lemma 16 that $N_{k / \mathbb{Q}}(w)=-1$ and $\operatorname{Tr}_{k / \mathbb{Q}}(w)= \pm 15$ or \pm 28 , that is, $w= \pm(15 \pm \sqrt{229}) / 2$ or $\pm(14 \pm \sqrt{197})$. If $w= \pm(15 \pm \sqrt{229}) / 2$, then $(u+27 v)=\left(w^{2}+27\right)=\mathfrak{p}^{3}$, where $\mathfrak{p}$ is a prime ideal of $\mathbb{Q}(\sqrt{229})$ dividing 19. Since $\mathfrak{p}$ is not principal, $u+27 v$ is not a cube in $\mathbb{Q}(\sqrt{229})$. (Note that the class number of $\mathbb{Q}(\sqrt{229})$ is 3.) If $w= \pm(14 \pm \sqrt{197})$, then $u+27 v$ is not a cube in $\mathbb{Q}(\sqrt{197})$, since $(u+27 v)=\left(2^{2} 7(15 \pm \sqrt{197})\right)=(2)^{3} \mathfrak{p}_{7}^{2} \mathfrak{p}_{7}^{\prime}$, where $(7)=\mathfrak{p}_{7} \mathfrak{p}_{7}^{\prime}$. 
If the sign is - , then

$$
\begin{aligned}
27 \operatorname{Tr}_{k / \mathbb{Q}}(w)^{2} & =\left\{-N_{k / \mathbb{Q}}(X)\right\}^{3}+730+54 N_{k / \mathbb{Q}}(w) \\
& = \begin{cases}\left\{-N_{k / \mathbb{Q}}(X)\right\}^{3}+784 & \text { if } N_{k / \mathbb{Q}}(w)=1, \\
\left\{-N_{k / \mathbb{Q}}(X)\right\}^{3}+676 & \text { if } N_{k / \mathbb{Q}}(w)=-1 .\end{cases}
\end{aligned}
$$

It follows from Lemma 16 that $N_{k / \mathbb{Q}}(w)=-1$ and $\operatorname{Tr}_{k / \mathbb{Q}}(w)= \pm 5$ or \pm 26 , that is, $w= \pm(13 \pm \sqrt{170})$ or $\pm(5 \pm \sqrt{29}) / 2$. If $w= \pm(13 \pm \sqrt{170})$, then $u+27 v$ is not a cube in $\mathbb{Q}(\sqrt{170})$, since $(u+27 v)=(26(12 \pm \sqrt{170}))=\mathfrak{p}_{2}^{3} \mathfrak{p}_{13}^{2} \mathfrak{p}_{13}^{\prime}$, where $(2)=\mathfrak{p}_{2}^{3},(13)=\mathfrak{p}_{13} \mathfrak{p}_{13}^{\prime}$. If $w= \pm(5 \pm \sqrt{29}) / 2$, then $u+27 v=v \varepsilon^{ \pm 2}$ $(\varepsilon=(5+\sqrt{29}) / 2)$. Thus, if $X^{3}=u+27 v$, then there exists $n \in \mathbb{Z}$ such that $v= \pm \varepsilon^{3 n-1}, X= \pm \varepsilon^{n-1}$, or $v= \pm \varepsilon^{3 n+1}, X= \pm \varepsilon^{n+1}$.

Remark. Lemma 17 is a generalization of Proposition 2.3 of [11] which states that the only $m \in \mathbb{Z}$ and $X \in \mathcal{O}_{\mathbb{Q}(\sqrt{29})}$ satisfying $X^{3}=\varepsilon^{4+12 m}-27 \varepsilon^{2}$ are $m=0$ and $X=-1$.

Proof of Proposition 15. Suppose that there exists an elliptic curve $E$ with properties stated in the proposition. We take $\Delta(E) \in \mathcal{O}_{k}^{\times}$. Letting

$$
(X, u, v)= \begin{cases}\left(c_{4}(E) /(t+3), \Delta(E), \Delta(E) / t\right) & \text { if }(t)=(1), \\ \left(3 c_{4}(E) /(t+3), 729 \Delta(E) / t, \Delta(E)\right) & \text { if }(t)=(729),\end{cases}
$$

we have $X^{3}=u+27 v, X \in \mathcal{O}_{k}, u, v \in \mathcal{O}_{k}^{\times}, u v= \pm \square$ by (3.3), (3.4) and Lemma $3(1)$. Hence, by Lemma 17 , we have $k=\mathbb{Q}(\sqrt{29}), u / v=-\varepsilon^{2},-\varepsilon^{\prime 2}$, where $\varepsilon=(5+\sqrt{29}) / 2$ is the fundamental unit of $\mathbb{Q}(\sqrt{29})$. If $(t)=(1)$, then $t=u / v=-\varepsilon^{2},-\varepsilon^{\prime 2}$, and $j(E)$ is equal to $J\left(-\varepsilon^{2}\right)=\left(\varepsilon^{2}-3\right)^{3} / \varepsilon^{4}$ or $J\left(-\varepsilon^{\prime 2}\right)=\left(\varepsilon^{\prime 2}-2\right)^{3} \varepsilon^{4}$. If $(t)=(729)$, then $t=729 v / u=-729 \varepsilon^{2},-729 \varepsilon^{\prime 2}$, and $j(E)$ is equal to $J\left(-729 \varepsilon^{2}\right)=-\left(1+216 \varepsilon^{\prime 2}\right)^{3} \varepsilon^{14}$ or $J\left(-729 \varepsilon^{\prime 2}\right)=$ $-\left(1+216 \varepsilon^{2}\right)^{3} \varepsilon^{\prime 14}$. Since the values of $j$-invariant obtained above are equal to $j\left(E_{5}\right), j\left(E_{5}^{\prime}\right), j\left(E_{6}^{\prime}\right)$ and $j\left(E_{6}\right)$ respectively, Lemma 14 implies our assertion.

Using Propositions 11, 12 and 15, we can give another proof of the following theorem which is the main theorem of [5]:

THEOREM 18. Up to isomorphism over $k=\mathbb{Q}(\sqrt{29})$, the only elliptic curves with everywhere good reduction over $k$ are $E_{5}, E_{5}^{\prime}, E_{6}$ and $E_{6}^{\prime}$.

Proof. Let $E$ be an elliptic curve with everywhere good reduction over $k=\mathbb{Q}(\sqrt{29})$ and let $\Delta(E) \in \mathcal{O}_{k}^{\times}$. Since $h_{k}^{(2)}=h_{k(\sqrt{ \pm \varepsilon})}^{(2)}=1, h_{k(\sqrt{-1})}^{(2)}=3$, and $E$ has no $k$-rational point of order 2 (see [1], [3]), we have $\Delta(E)=$ $-\varepsilon^{2 n}=-\square$. Since $h_{k}\left((3) \mathfrak{p}_{\infty}^{(1)} \mathfrak{p}_{\infty}^{(2)}\right)=2, h_{k(\sqrt[3]{\varepsilon})}\left((3) \mathfrak{P}_{\infty}^{(1)} \mathfrak{P}_{\infty}^{(2)}\right)=2$, and the prime number 3 is inert in $k$, we have by Propositions 11 and 12 that $j(E)$ is of the form $J(t),(t)=(1)$ or (729). Proposition 15 therefore implies that $E$ is isomorphic over $k$ to $E_{5}, E_{5}^{\prime}, E_{6}$ or $E_{6}^{\prime}$, as claimed. 


\section{References}

[1] S. Comalada, Elliptic curves with trivial conductor over quadratic fields, Pacific J. Math. 144 (1990), 237-258.

[2] J. E. Cremona, Algorithms for Modular Elliptic Curves, 2nd ed., Cambridge Univ. Press, 1997.

[3] H. Ishii, The non-existence of elliptic curves with everywhere good reduction over certain quadratic fields, Japan. J. Math. 12 (1986), 45-52.

[4] T. Kagawa, Determination of elliptic curves with everywhere good reduction over $\mathbb{Q}(\sqrt{37})$, Acta Arith. 83 (1998), 253-269.

[5] - Determination of elliptic curves with everywhere good reduction over real quadratic fields, Arch. Math. (Basel) 73 (1999), 25-32.

[6] M. Kida, Arithmetic of abelian varieties under field extensions, dissertation, Johns Hopkins Univ., 1994.

[7] -, Reduction of elliptic curves over certain real quadratic number fields, Math. Comp. 68 (1999), 1679-1685.

[8] —, Computing elliptic curves having good reduction everywhere over quadratic fields, preprint.

[9] M. Kida and T. Kagawa, Nonexistence of elliptic curves with good reduction everywhere over real quadratic fields, J. Number Theory 66 (1997), 201-210.

[10] H. Naito, On the Galois groups of the algebraic number fields generated by the 3-division points of elliptic curves, Mem. Fac. Ed. Kagawa Univ., II 36 (1986), $35-40$.

[11] T. Nakamura, On Shimura's elliptic curve over $\mathbb{Q}(\sqrt{29})$, J. Math. Soc. Japan 36 (1984), 701-707.

[12] R. G. E. Pinch, Elliptic curves over number fields, Ph.D. thesis, Oxford, 1982.

[13] - Elliptic curves with good reduction away from 3, Math. Proc. Cambridge Philos. Soc. 101 (1987), 451-459.

[14] J.-P. Serre, Propriétés galoisiennes des points d'ordre fini des courbes elliptiques, Invent. Math. 15 (1972), 259-331.

[15] B. Setzer, Elliptic curves over complex quadratic fields, Pacific J. Math. 74 (1978), 235-250.

[16] - Elliptic curves with good reduction everywhere over quadratic fields and having rational $j$-invariant, Illinois J. Math. 25 (1981), 233-245.

[17] J. H. Silverman, The Arithmetic of Elliptic Curves, Grad. Texts in Math. 106, Springer, 1986.

[18] J. Vélu, Isogénies entre courbes elliptiques, C. R. Acad. Sci. Paris 273 (1971), $238-241$.

Department of Mathematics

Ritsumeikan University

Kusatsu, Shiga 525-8577, Japan

E-mail: kagawa@se.ritsumei.ac.jp 